ISSN 1516-635X Oct - Dec 2015 / Special Issue

Nutrition - Poultry feeding additives / 023-030

http://dx.doi.org/10.1590/1516-635xSpeciallssue Nutrition-PoultryFeedingAdditives023-030

\section{Effects of Different Dietary Rosmarinus Officinalis Powder and Vitamin E Levels on the Performance and Gut Gross Morphometry of Broiler Chickens}

\section{-Author(s)}

\section{Rostami $\mathrm{H}^{\prime}$}

Seidavi $A^{\prime}$

Dadashbeiki M"

Asadpour YII

Simões JV

Department of Animal Science, Rash Branch, Islamic Azad University, Rasht, Iran.

" Department of Veterinary Science, Rasht Branch, Islamic Azad University, Rasht, Iran.

III Agricultural and Natural ResourcesResearch Centerof Guilan, Rasht, Iran.

IV Department of Veterinary Science, University of Trás-os-Montes e Alto Douro. Quinta de Prados 5000-811 Vila Real, Portugal.

\section{Mail Address}

Corresponding author e-mail address Alireza Seidavi

Department of Animal Science, Rasht Branch, Islamic Azad University, Rasht,

Iran, Zip code: 4185743999

Tel: (+989) 113313073

E-mail: alirezaseidavi@iaurasht.ac.ir

\section{EKeywords}

Gut, nutrition, rosemary, vitamin $\mathrm{E}$, weight gain.

\section{ABSTRACT}

The main objective of the present study was to evaluate the effects of the dietary addition of different levels of rosemary powder (RP) and vitamin $\mathrm{E}$ (VitE) on broiler performance and gut gross morphometry. A total of 270 one-day-old Ross 308 male chicks were randomly assigned to nine dietary treatments with three replicates of 10 birds each. Treatments consisted of diets were supplemented with $0,0.5$ or $1.0 \% \mathrm{RP}$ and 0,100 or $200 \mathrm{mg} / \mathrm{kg}$ VitE (alpha-tocopherol acetate). Feed intake and weight gain were recorded weekly. On day 42, one bird per replicate was euthanized after blood sampling. Gastrointestinal tract segments were measured and/or weighed. Means were compared by least significant difference. Overall, broilers fed1.0\% RP presented lower $(p<0.05)$ feed intake, weight gain, and final weight body than those fed 0.5\% RP. Broilers fed the 0.5\% RP plus $200 \mathrm{mg} / \mathrm{kg}$ VitE diet presented higher weight gain $(p<0.05)$ in than those in the control group ( $0 \% \mathrm{RP}$ and $0 \mathrm{mg} \mathrm{VitE).} \mathrm{Jejunum} \mathrm{length} \mathrm{and} \mathrm{weight,} \mathrm{colon}$ length and width, and right cecum weight were also negatively affected $(p<0.05)$ by the $1.0 \%$ RP diet when compared with the 0.5\% RP diet. We suggest that the dietary supplementation of $0.5 \%$ RP plus $200 \mathrm{mg} /$ $\mathrm{kg}$ of vitE improves broiler performance and does not have significant adverse effects on gross gut morphometry. Dietary VitE may play a potential protective role against the negative effects of high levels of RP.

\section{INTRODUCTION}

During the last decades several additives with probiotic, prebiotic or symbiotic activity have been evaluated as a replacement of antimicrobials as growth promoters in intensive poultry production. Moreover, research on the use of natural plants with medicinal and/or aromatic proprieties as phytogenic feed additives have been carried out. These studies have shown that not only they improve broiler performance and health, and consequently animal welfare, but also to benefits food quality for human consumption, the environment, and public health (Windisch et al., 2008; Franz et al., 2010; Hashemi \& Davoodi, 2011; Charles, 2013).

Rosemary (Rosmarinus officinalis L.) is an aromatic has been studied due to the antioxidant effects (Moreno et al., 2006) of some of its active compounds, such as carnosol, rosmanol and their acid forms or flavonoids (Ibañez et al., 2003), on bird physiology. Several studies reported beneficial effects of rosemary powder (RP) or their extracts (essential oils) on broilers performance, hematological and biochemical parameters, immune status, and meat or egg quality (Hernández et al., 2004; Ghazalah \& Ali, 2008; Polat et al., 2011; Yesilbag et al., 2011, 2012, 2013). Some of these studies (Ghazalah \& Ali, 2008; Polat et al., 2011; Yesilbag et al., 2011) also describe negative effects on performance, at least during short starter or finisher periods, and on hematological parameters during the 6-7 weeks production cycle, 
when the RP increases in diet from low to high levels. Nonetheless, due to potential dual effects on broiler performance, more detailed evaluation, using different inclusion levels, is essential for the effective use of RP as feed additive in broilers.

Although no effects of RP on intestinal weight or their annex glands were observed by Hernández et al. (2004), Ghazalah \& Ali (2008) reported a negative effect on broiler gizzard weight. However, effects of other feed additives, such as garlic, oregano, cinnamon, pepper, thyme and fermented plant products (rice bran and fermented fruit and vegetables) on intestinal histology were observed (García et al., 2007; Abdullah et al., 2010; Lokaewmanee et al., 2012; Giannenas et al., 2014). In fact, more research is need to determine if RP as a feed additive causes gross morphometric changes in the digestive system, particularly on weight, villus density, and on the enterocytes (Applegate et al., 1999).

On other hand, Vitamin E (VitE) is recognized as an elective and natural antioxidant at cell membrane level. It is essential for the health of animals submitted to stressful rearing conditions, and it used as a feed additive in broilers diets (Khan et al., 2012; Lu et al. 2014a, 2014b). However, Xiao et al. (2013) suggested that there are functional redundancies between VitE and other natural compounds in broiler diets. Therefore is central to detect the effects of the use of different levels of RP and VitE and their interactions as feed additive on broiler performance and development.

The main objective of this study was to determine the effect of different dietary levels of RP and vitE on the performance to broilers during a 6-week period and on their digestive system developmentat42 days of age.

\section{MATERIALS AND METHODS}

\section{Birds and housing}

The experiment was conducted for 42 days in a poultry house of Agricultural and Natural Resources Research Centerof Guilan, Guilan, Rasht, Iran. Before and after to the experiment, the facility was thoroughly cleaned and disinfected, including all drinkers and feeders. Care was taken to minimize the number of animals used. All procedures have been approved by the authors' Institution Ethic Committee.

In total, 270 one-day-old Ross 308 male chicks (Aviagen, Newbridge, Scotland, UK 35805) were housed in a $5 \times 20 \mathrm{mbroiler}$ house equipped with a potent fan (1400 $\mathrm{m}^{3}$ per hour) and five smaller fans (total of $3500 \mathrm{~m}^{3}$ per hour). Heat was generated by a heater programed according to the Ross 308 manual (Aviagen, Newbridge, Scotland, UK 35805; infoworldwide@aviagen.com). Air humidity was maintained at 55 to $65 \%$ during the starter growing period by spraying water on the floor. Artificial lighting was provided by 20-watt lamps were installed $2.2 \mathrm{~m}$ above the floor for three hours per day, between 19:00 and 22:00, until day 42 .

Sanitation principles and health measures for chicken production were applied. Drinkers were cleaned and washed daily. Birds were vaccinated against infectious bursal disease, avian influenza, infectious bronchitis, and Newcastle disease. After each vaccination, 1:1000 multivitamin + electrolytes solution was mixed in the drinking water for 24 hours.

\section{Treatments and diet composition}

Nine treatments with three replicates of 10 birds, with similar body weight ( $p>0.05)$, were applied. All birds were fed according to the producer's feeding instructions. The mean composition of basal diets and their nutrient composition for the starter (1-21 days of age) and finisher (22-42 days of age) periods are given in Table 1.

Rosemary powder (RP) was included at levels of 0.5 or $1.0 \%$ in the basal diet, and VitE at levels of and 0,100 , or $200 \mathrm{mg} / \mathrm{kg}$ of VitE, according to each treatment: control group T1 (RPO + VitE0), T2 (RPO + VitE100), T3 (RPO + VitE200), T4 (RP0.5 + VitE0), T5 (RP0.5 + VitE100), T6 (RP0.5 + VitE200), T7 (RP1.0 + VitE0), T8 (RP1.0 + VitE100), and T9 (RP1.0 + VitE200).

Rosemary nutritional levels were analyzed according to AOAC methods (AOAC, 2005), and presented was $3124 \mathrm{kcal}$ gross energy/kg, $2511 \mathrm{kcal}$ metabolizable energy $/ \mathrm{kg}(\mathrm{ME}=0.80378 * \mathrm{GE}), 4.36 \%$ crude protein, and $20.73 \%$ crude fiber. However, due to relatively low doses of RP, of up to $10 \mathrm{~g}$ per $\mathrm{kg}$ of feed, RP nutritional levels were not considered during basal diet formulation.

Feed remaining in feeders was weighed and removed at the end of each week. This feed was not taken in account for intake calculation.

\section{Performance, hematology and carcass parameters}

Feed intake (g) and weight gain (g) were recorded weekly. Feed conversion ratio (FCR $=$ total feed intake / total body weight gain) and production index [PI = total body weight $x$ livability (\%) / (age days $x$ feed conversion ratio $\times 10)$ ] were calculated. 
Table 1 - Feed ingredients and calculated nutrient composition of the basal starter (1-21 days of age) and finisher (22-42 days of age) diets.

\begin{tabular}{|c|c|c|c|c|c|}
\hline \multicolumn{6}{|l|}{ Diet } \\
\hline Ingredient (g/kg) & Starter diet & Finisher diet & Calculated composition & Starter diet & Finisher diet \\
\hline Corn & 556.3 & 585.9 & Phosphorus (\%) & 0.74 & 0.69 \\
\hline Soybean oil & 27.4 & 43.7 & Available Phosphorus (\%) & 0.50 & 0.45 \\
\hline $\mathrm{NaHCO}_{3}$ & 1.9 & 1.2 & Potassium (\%) & 0.90 & 0.84 \\
\hline Soybean meal & 355.3 & 322.3 & Manganese (mg/kg) & 120.00 & 120.00 \\
\hline Gluten meal & 10 & 0 & Sodium (\%) & 0.16 & 0.16 \\
\hline Wheat bran & 7 & 10 & Glycine (\%) & 0.93 & 0.86 \\
\hline $\mathrm{CaCO} 3$ & 12.9 & 10.5 & Serine (\%) & 1.10 & 1.02 \\
\hline Сa\%22P\%18 & 18.9 & 16.7 & Gly+Ser (\%) & 2.40 & 2.21 \\
\hline $\mathrm{NaCl}$ & 2.3 & 2.7 & Histidine (\%) & 0.59 & 0.55 \\
\hline Mineral Mixture ${ }^{1}$ & 3 & 3 & Isoleucine (\%) & 0.85 & 0.85 \\
\hline Vitamin Mixture ${ }^{2}$ & 3 & 3 & Leucine (\%) & 1.90 & 1.80 \\
\hline DL-Methionine & 1.2 & 1 & Lysine (\%) & 1.27 & 1.11 \\
\hline Lysine-Hydro-Chloride & 0.8 & 0 & Methionine (\%) & 0.47 & 0.42 \\
\hline Total & 1000 & 1000 & Met+Cys (\%) & 0.85 & 0.76 \\
\hline Calculated composition & & & Phenylalanine (\%) & 1.08 & 0.98 \\
\hline Dry Matter (\%) & 86.47 & 87.08 & Tyrosine (\%) & 0.89 & 0.81 \\
\hline Energy (ME) (kcal/kg) & 3025 & 3150 & Phe+Tyr (\%) & 1.97 & 1.79 \\
\hline Crude Protein (\%) & 23.00 & 21.00 & Threonine (\%) & 0.83 & 0.78 \\
\hline Ether Extract (\%) & 5.25 & 6.95 & Tryptophan (\%) & 0.3 & 0.28 \\
\hline Linoleic Acid (\%) & 2.77 & 3.66 & Valine (\%) & 1.00 & 0.96 \\
\hline Crude Fiber (\%) & 2.69 & 2.66 & Arginine (\%) & 1.30 & 1.30 \\
\hline Calcium (\%) & 1.05 & 0.90 & & & \\
\hline
\end{tabular}

${ }^{1}$ Cu: $3 \mathrm{mg} / \mathrm{g} ; \mathrm{Zn}: 15 \mathrm{mg} / \mathrm{g}$; Mn: $20 \mathrm{mg} / \mathrm{g}$; Fe: $10 \mathrm{mg} / \mathrm{g} ; \mathrm{K}: 0.3 \mathrm{mg} / \mathrm{g}$

${ }^{2}$ Vitamin A: 5000 IU/g; Vitamin D3: 500 IU/g; Vitamin E: 3 mg/g; Vitamin K3: 1.5 mg/g; Vitamin B6: 13 mg/g; Vitamin B2: 1 mg/g; Calcium Pantothenate: 4 mg/g; Niacin: 15 mg/g

Before blood collection, feed was removed from feeders for a period of four hours in an attempt to allow stabilization of the various plasma components. Blood was collected in the morning to further reduce the variability of the plasma components to be measured. At 42 days of age, a $5 \mathrm{~mL}$ volume of venous blood was collected from the ulnar vein of the wing of one bird per replicate. Care was taken to choose the most representative birds with respect to body weight compared with the average body weight of their group. The whole blood sample was transferred from the syringe into a tube coated with $10 \mathrm{mg}$ of the anticoagulante thylene diaminetetra acetic acid (EDTA). Blood samples werecentrifuged at 3000 rpm for 20 min to ensure the separation of blood cells from plasma. Plasma was collected and stored at $-20^{\circ} \mathrm{C}$ until further analyses. Plasma component analyses were based on standard protocols, using the Roche Cobas Integra 400 Plus autoanalyzer (Roche Diagnostics, GmbH, Mannheim, Germany). Uric acid, total protein, total cholesterol, triglycerides, low density lipoprotein (LDL) cholesterol, high density lipoprotein(HDL) cholesterol, very high density lipoproteins (VLDL), aspartate amino transferase (AST) and alanine amino transferase (ALT) were assayed using commercial kits (Teif Azmoon Pars, Co., Tehran, Iran).

On day 42, the most representative bird from each replicate was euthanized after 4 hours of fasting for complete evacuation of the gut. After blood sample collection, these birds were used for measuring gastrointestinal tract characteristics and carcass yield. Birds were fully plucked by dry plucking method. Feet were separated from the carcass by the tibiotarsal joint. Neck, wingtips, gut, and liver were removed and the empty or edible carcass was weighed and intestinal segments dimensions were recorded.

Carcass parts were dissected and separately weighed. Parts included the head, breast, wings, femurs, abdominal fat, pancreas, gizzard, crop, lungs, heart, liver, kidneys, and digestive tract. Length, width, and wall thickness of the different gut segments were also recorded. Head, breast, abdominal fat, pancreas, full gizzard, full crop, lung, heart, liver, kidney, brain, testicles, duodenum, ileum, jejunum, colon, left and right cecum, vertebral column with neck and proventriculus were weighed.

All abdominal fat, including that around the rectum, gizzard and proventriculus, was collected and weighed. 
The length $(\mathrm{cm})$, width $(\mathrm{mm})$ and wall thickness $(\mathrm{mm})$ of duodenum, ileum, jejunum, left and right cecum, and colon were recorded.

Total weight of all dissected parts and the weights of various segments of the digestive tract were calculated relative to eviscerated carcass weight according to the following formula: [(weight of component/eviscerated carcass weight $) \times 100]$.

\section{Statistical analysis}

Data were submitted to two-way analysis of variance, according to a $3 \times 3$ factorial arrangement, with three RP inclusion levels $(0,0.5$, and $1.0 \%$ in diet) and three vitE inclusion levels $(0,100$, and $20 \mathrm{mg} / \mathrm{kg}$ in diet). Data were analyzed using the GLM procedure of SPSS (1997) statistical software. The means ( \pm SEM) were compared by least significant difference (LSD) test. The results were considered different at 5\% significance level.

\section{RESULTS}

\section{Performance}

Performance results are reported in Tables 2, 3, and 4 for the starter (1-21 days of age), finisher (22-42 days of age) and entire (1-42 days of age) experimental periods, respectively.

Overall, a deterioration effect $(p<0.05)$ on the feed intake, weight gain, feed conversion ratio or production index were observed between groups when rosemary inclusion levels increased from 0 or 0.5 to
$1.0 \%$. These findings were obvious during both feed periods. During the starter period, adverse effects of the supplementation of $1 \%$ RP alone on FCR, weight gain, and production index were observed $(p<0.05)$. During the finisher period, this deleterious effect was observed on feed intake and weight gain as RP increased $(p<0.05)$ from 0.5 to $1 \%$.

Average weight gain during the entire rearing period was negatively affected $(p<0.05)$ by $1 \%$ RP addition compared with the level of $0.5 \%$. The supplementation of $200 \mathrm{mg}$ VitE alone increased weight gain during finisher period $(p<0.05)$, but not during the starter period ( $p>0.05$ ).

There was no effect of the supplementation of different combinations of RP and VitE on the evaluated performance parameters.

\section{Hematological parameters}

Uric acid and total protein levels were significantly affected $(p<0.05)$ by RP and VitE additives (Table 5). However, total cholesterol (129.0 $\pm 8.9 \mathrm{mg} / \mathrm{dL})$, triglyceride $(68.0 \pm 19.4 \mathrm{mg} / \mathrm{dL}), \mathrm{VLDL}(13.7 \pm 3.9$ $\mathrm{mg} / \mathrm{dL}), \mathrm{HDL}$ cholesterol $(82.3 \pm 11.2 \mathrm{mg} / \mathrm{dL})$, and LDL cholesterol $(33.0 \pm 7.8 \mathrm{mg} / \mathrm{dL})$ levels, $\mathrm{LDL} / \mathrm{HDL}$ ratio $(0.49 \pm 0.12)$, and AST $(280.0 \pm 41.2 \mathrm{UI} / \mathrm{L})$ and $\operatorname{ALT}(2.7$ $\pm 0.6 \mathrm{UI} / \mathrm{L})$ were similar $(\mathrm{p}>0.05)$ among groups.

\section{Gut gross morphometry and carcass characteristics}

All significant differences in duodenal, jejunal, and colonic morphometry are reported in Table 6.

Table 2 - Performance (mean) of Ross 308 broilers from 1-21 days of age (starter period).

\begin{tabular}{|c|c|c|c|c|c|}
\hline \multirow[b]{2}{*}{ Treatment } & & \multicolumn{4}{|c|}{ Trait } \\
\hline & & Feed intake (g) & Feed conversion ratio & Weight gain (g) & Production index \\
\hline \multirow{3}{*}{ Rosemary (\% in diet) } & 0 & 1199.1 & $1.37^{a}$ & $877.8^{a}$ & $301.4^{a}$ \\
\hline & 0.5 & 1188.6 & $1.39^{a, b}$ & $857.7^{a, b}$ & $296.1^{a, b}$ \\
\hline & 1.0 & 1190.7 & $1.42^{b}$ & $837.9^{b}$ & $274.5^{b}$ \\
\hline \multirow{3}{*}{ Vitamin E (mg/kg) } & 0 & 1188.6 & 1.36 & 871.5 & 298.0 \\
\hline & 100 & 1188.6 & 1.38 & 863.1 & 289.1 \\
\hline & 200 & 1203.3 & 1.40 & 858.9 & 285.0 \\
\hline RP X VitE interaction & & $p<0.05$ & N.S. & N.S. & N.S. \\
\hline T1: RPO + VitEO & & $1201.2^{\mathrm{ab}}$ & 1.33 & 900.9 & 315.4 \\
\hline T2: RPO + VitE100 & & $1197.0^{\mathrm{ab}}$ & 1.38 & 865.8 & 294.1 \\
\hline T3: RPO + VitE200 & & $1203.3^{\mathrm{ab}}$ & 1.39 & 867.7 & 294.7 \\
\hline T4: RPO. 5 + VitEO & & $1194.9^{a b}$ & 1.37 & 869.4 & 298.6 \\
\hline T5: RP0.5 + VitE100 & & $1184.4^{\mathrm{ab}}$ & 1.35 & 879.1 & 295.3 \\
\hline T6: RP0.5 + VitE200 & & $1188.6^{\mathrm{ab}}$ & 1.35 & 880.7 & 294.2 \\
\hline T7: RP1.0 + VitE0 & & $1167.6^{\mathrm{a}}$ & 1.39 & 840.8 & 280.0 \\
\hline T8: RP1.0 + VitE100 & & $1186.5^{\mathrm{ab}}$ & 1.41 & 842.3 & 277.8 \\
\hline T9: RP1.0 + VitE200 & & $1220.1^{b}$ & 1.47 & 827.6 & 265.7 \\
\hline
\end{tabular}

$a, b$ : Different letters within the same column indicate significant differences among treatment groups $(p<0.05)$.

N.S. - Not significant. 
Table 3 - Performance (mean) of Ross 308 broilers from 22-42 days of age (finisher period).

\begin{tabular}{|c|c|c|c|c|c|}
\hline \multirow[b]{2}{*}{ Treatment } & & \multicolumn{4}{|c|}{ Trait } \\
\hline & & Feed intake (g) & Feed conversion ratio & Weight gain (g) & Production index \\
\hline \multirow{3}{*}{ Rosemary (\% in diet) } & 0 & $3410.4^{\mathrm{ab}}$ & 2.00 & $1706.3^{\mathrm{ab}}$ & 385.6 \\
\hline & 0.5 & $3555.3^{a}$ & 1.95 & $1826.1^{\mathrm{a}}$ & 442.9 \\
\hline & 1.0 & $3355.8^{b}$ & 2.09 & $1701.8^{b}$ & 401.7 \\
\hline \multirow{3}{*}{ Vitamin E (mg/kg) } & 0 & 3393.6 & 2.02 & $1684.0^{\mathrm{a}}$ & $384.4^{a}$ \\
\hline & 100 & 3427.2 & 2.00 & $1717.2^{\mathrm{ab}}$ & $393.8^{\mathrm{ab}}$ \\
\hline & 200 & 3504.9 & 1.91 & $1832.3^{b}$ & $452.1^{b}$ \\
\hline RP X VitE interaction & & N.S. & N.S. & N.S. & N.S. \\
\hline T1: RPO + VitEO & & 3389.4 & 2.03 & 1673.7 & 368.9 \\
\hline T2: RPO + VitE100 & & 3347.4 & 2.07 & 1618.0 & 341.6 \\
\hline T3: RPO + VitE200 & & 3498.6 & 1.91 & 1827.7 & 446.3 \\
\hline T4: RPO.5 + VitE0 & & 3502.8 & 2.00 & 1753.6 & 413.5 \\
\hline T5: RP0.5 + VitE100 & & 3559.5 & 1.96 & 1814.8 & 436.3 \\
\hline T6: RPO.5 + VitE200 & & 3605.7 & 1.89 & 1907.0 & 479.0 \\
\hline T7: RP1.0 + VitEO & & 3282.5 & 2.02 & 1622.5 & 370.7 \\
\hline T8: RP1.0 + VitE100 & & 3372.6 & 1.96 & 1720.5 & 403.5 \\
\hline T9: RP1.0 + VitE200 & & 3410.4 & 1.94 & 1762.2 & 431.0 \\
\hline
\end{tabular}

a,b: Different letters within the same column indicate significant differences among treatment groups $(p<0.05)$.

N.S. - Not significant.

Table 4 - Performance (mean) of Ross 308 broilers from 1-42 days of age fed diets containing the different levels of rosemary powder and vitamin $\mathrm{E}$.

\begin{tabular}{|c|c|c|c|c|c|c|}
\hline \multirow{2}{*}{ Treatment } & & \multicolumn{5}{|c|}{ Trait } \\
\hline & & Feed intake (g) & Feed conversion ratio & Weight gain (g) & Weight chick at $42^{\text {nd }}$ day (g) & Production index \\
\hline \multirow{3}{*}{ Rosemary (\% in diet) } & 0 & $4611.6^{\mathrm{ab}}$ & 1.79 & $2583.0^{\mathrm{ab}}$ & $2638.9^{\mathrm{ab}}$ & 357.4 \\
\hline & 0.5 & $4746.0^{a}$ & 1.76 & $2704.8^{a}$ & $2751.1^{\mathrm{a}}$ & 388.0 \\
\hline & 1.0 & $4548.6^{b}$ & 1.79 & $2536.8^{b}$ & $2586.9^{b}$ & 357.3 \\
\hline \multirow{3}{*}{ Vitamin E (mg/kg) } & 0 & 4582.2 & 1.80 & $2543.6^{a}$ & $2608.3^{a}$ & 355.8 \\
\hline & 100 & 4615.8 & 1.79 & $2578.8^{\mathrm{ab}}$ & $2628.6^{\mathrm{ab}}$ & 356.7 \\
\hline & 200 & 4708.2 & 1.75 & $2692.2^{\mathrm{b}}$ & $2740.0^{b}$ & 387.2 \\
\hline \multicolumn{2}{|l|}{ RP X VitE interaction } & N.S. & N.S. & N.S. & N.S. & N.S. \\
\hline \multicolumn{2}{|l|}{ T1: RPO + VitE0 } & 4590.6 & 1.78 & 2574.6 & 2640.3 & 354.5 \\
\hline \multicolumn{2}{|l|}{ T2: RP0 + VitE100 } & 4544.4 & 1.83 & 2482.2 & 2532.3 & 328.6 \\
\hline \multicolumn{2}{|l|}{ T3: RPO + VitE200 } & 4699.8 & 1.74 & 2696.4 & 2744.0 & 389.0 \\
\hline \multicolumn{2}{|l|}{ T4: RPO.5 + VitEO } & 4741.8 & 1.81 & 2625.0 & 2674.2 & 373.3 \\
\hline \multicolumn{2}{|l|}{ T5: RP0.5 + VitE100 } & 4792.2 & 1.78 & 2692.2 & 2742.7 & 384.0 \\
\hline \multicolumn{2}{|l|}{ T6: RP0.5 + VitE200 } & 4456.2 & 1.60 & 2788.8 & 2836.3 & 406.6 \\
\hline \multicolumn{2}{|l|}{ T7: RP1.0 + VitE0 } & 4561.2 & 1.85 & 2465.4 & 2510.4 & 339.7 \\
\hline \multicolumn{2}{|l|}{ T8: RP1.0 + VitE100 } & 4628.4 & 1.81 & 2562.0 & 2610.8 & 357.4 \\
\hline \multicolumn{2}{|l|}{ T9: RP1.0 + VitE200 } & 4741.8 & 1.83 & 2591.4 & 2639.6 & 366.0 \\
\hline
\end{tabular}

a,b: Different letters within the same column indicate significant differences among treatment groups $(p<0.05)$.

N.S. - Not significant.

Dietary RP addition influenced $(p<0.05)$ jejunum weight (0\% RP: $75.2 \mathrm{~g}$ vs. $0.5 \%$ RP: $54.3 \mathrm{~g}$ or $1.0 \%$ RP: $55.9 \pm 5.0 \mathrm{~g})$, jejunum relative weight (0\% RP: $3.00 \% \mathrm{~mm}$ vs. $0.5 \% \mathrm{RP}: 2.19 \pm 0.20 \mathrm{~g}$ ), jejunum length (0\% RP: $1291.1 \mathrm{~cm}$ vs. $1.0 \%$ RP: $1112.2 \pm 45.5$ $\mathrm{cm}$ ), colon length (0\% RP: $94.4 \mathrm{~cm}$ vs. $1.0 \%$ RP: 76.1 $\pm 3.2 \mathrm{~cm}$ ), colon width (0\% RP: $6.3 \mathrm{~mm}$ vs. $1.0 \%$ RP: $4.7 \pm 0.4 \mathrm{~mm})$, right cecum absolute weight ( $0.5 \% \mathrm{RP}$ : $8.3 \mathrm{~g}$ vs. $1.0 \%$ RP: $6.3 \pm 0.5 \mathrm{~g} ; \mathrm{p}<0.05)$ and relative weight (0\% RP: $0.26 \%$ vs. $0.5 \%$ RP: $0.34 \pm 0.02 \%$ ), but no significant RP $\times$ VitE interactions were observed for these traits.

The duodenum width was influenced by RP (0\% RP: $5.6 \mathrm{~mm}$ or $0.5 \%$ RP: $5.3 \mathrm{~mm}$ vs. $1.0 \%$ RP: $6.6 \pm 0.3$ $\mathrm{mm} ; \mathrm{p}<0.05)$, Vite (100g VitE: $5.2 \mathrm{~mm}$ vs. $200 \mathrm{~g}$ VitE: $6.6 \pm 0.3 \mathrm{~mm} ; \mathrm{p}<0.05)$ and by RP $\times$ VitE interactions $(p<0.01)$. Higher duodenum width $(p<0.01)$ was observed in control group $(\mathrm{T} 1 ; 7.0 \pm 0.5 \mathrm{~mm})$ compared with T2 $(4.2 \mathrm{~mm})$ and T4 (4.6 mm). 
Table 5 - Plasma uric acid and total protein levels (mean) of 42-d-old Ross 308 broilers fed diets containing the different levels of rosemary powder and Vitamin E.

\begin{tabular}{lcll}
\hline \multirow{2}{*}{ Treatment } & & \multicolumn{2}{c}{ Trait } \\
\cline { 2 - 4 } & & Uric acid $(\mathrm{mg} / \mathrm{dL})$ & Total protein $(\mathrm{g} / \mathrm{dL})$ \\
& 0 & $4.4^{\mathrm{ab}}$ & 3.8 \\
Rosemary (\% in diet) & 0.5 & $3.9^{\mathrm{a}}$ & 3.6 \\
& 1.0 & $5.8^{\mathrm{b}}$ & 4.0 \\
\hline & 0 & $5.7^{\mathrm{a}}$ & 4.0 \\
\hline & 100 & $3.9^{\mathrm{b}}$ & 3.7 \\
\hline Vitamin E (mg/kg) & 200 & $4.6^{\mathrm{ab}}$ & 3.7 \\
\hline RP X VitE interaction & & N.S. & $\mathrm{p}=0.05$ \\
\hline T1: RPO + VitE0 & & 5.3 & $3.9^{\mathrm{ab}}$ \\
\hline T2: RP0 + VitE100 & & 3.7 & $4.1^{\mathrm{ab}}$ \\
\hline T3: RP0 + VitE200 & & 4.3 & $3.4^{\mathrm{a}}$ \\
\hline T4: RP0.5 + VitE0 & & 4.3 & $3.8^{\mathrm{ab}}$ \\
\hline T5: RP0.5 + VitE100 & & 3.8 & $3.3^{\mathrm{a}}$ \\
\hline T6: RP0.5 + VitE200 & & 3.7 & $3.8^{\mathrm{ab}}$ \\
\hline T7: RP1.0 + VitE0 & & 7.6 & $4.4^{\mathrm{b}}$ \\
\hline T8: RP1.0 + VitE100 & & 4.1 & $3.6^{\mathrm{ab}}$ \\
\hline T9: RP1.0 + VitE200 & & 5.7 & $4.0^{\mathrm{ab}}$ \\
\hline
\end{tabular}

$a, b$ : Different letters within the same column indicate significant differences among treatment groups $(p<0.05)$.

N.S. - Not significant.

The weight of the remaining carcass parts and of other organs or tissues evaluated in the present study was not influenced ( $p>0.05$ ) by the treatments and no significant RP $\times$ VitE interactions were observed either (p>0.05).

\section{DISCUSSION}

In the present study, there were both positive and negative effects of RP and VitE feed additives on several performance parameters, according the added levels or their interactions. Overall, the dietary addition of $0.5 \%$ RP promoted in higher feed intake and weight gain at 42 days compared with $1.0 \%$ RP; however, the obtained values were not different from those obtained in the control group. This indicates that the dietary addition of $0.5 \%$ RP did not improve broiler performance and that the level of 1.0\% RP negatively affected performance. Ghazalah \& Ali (2008) observed positive effects of the dietary addition of $0.5 \%$ rosemary leaves on the weight gain, body weight, and feed conversion ratio of 49 -d-old broilers compared with the control group. However, those authors also observed lower live body weight and feed intake when 1 and $2 \%$ of rosemary leaves were added to the diet and attributed this effect to a reduction in feed palatability for young chicks.

On other hand, the supplementation of $200 \mathrm{mg}$ VitE/kg had positive effect on the weight gain and feed intake on day 42. This suggests that $200 \mathrm{mg}$ VitE/kg diet may improve broiler performance. In addition, the significant interaction between that VitE level counteracted the negative effects of aflatoxin when added in combination with 1.0\% RP: during

Table 6 - Duodenal, jejunal, and colonic width, weight, and length (mean) of 42-d-old Ross 308 broilers fed diets containing the different levels of rosemary powder and Vitamin $\mathrm{E}$.

\begin{tabular}{|c|c|c|c|c|c|c|c|c|c|}
\hline \multirow{2}{*}{ Treatment } & & \multicolumn{8}{|c|}{ Trait } \\
\hline & & $\begin{array}{l}\text { Duodenum } \\
\text { width (mm) }\end{array}$ & $\begin{array}{l}\text { Jejunum } \\
\text { weight (g) }\end{array}$ & $\begin{array}{c}\text { Jejunum } \\
\text { Relative } \\
\text { weight (\%) }\end{array}$ & $\begin{array}{l}\text { Jejunum length } \\
(\mathrm{cm})\end{array}$ & $\begin{array}{l}\text { Colon length } \\
(\mathrm{cm})\end{array}$ & $\begin{array}{l}\text { Colon width } \\
\text { (mm) }\end{array}$ & $\begin{array}{l}\text { Right cecum } \\
\text { weight }(\mathrm{g})\end{array}$ & $\begin{array}{l}\text { Right cecum } \\
\text { relative } \\
\text { weight (\%) }\end{array}$ \\
\hline \multirow{3}{*}{$\begin{array}{l}\text { Rosemary } \\
\text { (\% in diet) }\end{array}$} & 0 & $5.6^{a}$ & $75.2^{\mathrm{a}}$ & $3.00^{\mathrm{a}}$ & $1291.1^{\mathrm{a}}$ & $94.4^{\mathrm{a}}$ & $6.3^{\mathrm{a}}$ & 6.5 & $0.26^{a}$ \\
\hline & 0.5 & $5.3^{\mathrm{a}}$ & $54.3^{b}$ & $2.19^{b}$ & $1183.3^{\mathrm{ab}}$ & $87.2^{\mathrm{ab}}$ & $4.9^{a b}$ & $8.3^{a}$ & $0.34^{\mathrm{b}}$ \\
\hline & 1.0 & $6.6^{b}$ & $55.9^{b}$ & $2.41^{\mathrm{ab}}$ & $1112.2^{b}$ & $76.1^{\mathrm{b}}$ & $4.7^{b}$ & $6.3^{b}$ & 0.27 \\
\hline \multirow{3}{*}{$\begin{array}{l}\text { Vitamin E (mg/ } \\
\mathrm{kg} \text { ) }\end{array}$} & 0 & $6.0^{\mathrm{ab}}$ & 66.7 & 2.80 & 1225.6 & 85.6 & 5.9 & 6.9 & 0.29 \\
\hline & 100 & $5.2^{\mathrm{a}}$ & 61.1 & 2.49 & 1251.1 & 87.2 & 4.8 & 7.7 & 0.31 \\
\hline & 200 & $6.3^{b}$ & 57.5 & 2.35 & 1110.0 & 85.0 & 5.1 & 6.4 & 0.27 \\
\hline \multicolumn{2}{|c|}{ RP $\times$ VitE interaction } & $p<0.01$ & N.S. & N.S. & N.S. & N.S. & N.S. & N.S. & N.S. \\
\hline \multicolumn{2}{|l|}{ T1: RPO + VitEO } & $7.0^{\mathrm{a}}$ & 81.5 & 3.37 & 1283.3 & 90.0 & 7.2 & 6.1 & 0.25 \\
\hline \multicolumn{2}{|c|}{ T2: RPO + VitE100 } & $4.2^{b}$ & 69.3 & 2.87 & 1366.7 & 100.0 & 5.6 & 7.9 & 0.33 \\
\hline \multicolumn{2}{|c|}{ T3: RPO + VitE200 } & $5.6^{\mathrm{abc}}$ & 74.7 & 2.90 & 1223.3 & 93.3 & 6.0 & 5.4 & 0.21 \\
\hline \multicolumn{2}{|c|}{ T4: RPO.5 + VitEO } & $4.6^{b, c}$ & 52.5 & 2.17 & 1176.7 & 83.3 & 5.4 & 9.4 & 0.39 \\
\hline \multicolumn{2}{|c|}{ T5: RP0.5 + VitE100 } & $4.8^{\mathrm{abc}}$ & 58.0 & 2.26 & 1256.7 & 85.0 & 4.7 & 6.8 & 0.26 \\
\hline \multicolumn{2}{|c|}{ T6: RP0.5 + VitE200 } & $6.4^{\mathrm{abc}}$ & 52.2 & 2.12 & 1116.7 & 93.3 & 4.8 & 8.7 & 0.36 \\
\hline \multicolumn{2}{|c|}{ T7: RP1.0 + VitE0 } & $6.4^{\mathrm{abc}}$ & 66.1 & 2.86 & 1216.7 & 83.3 & 5.3 & 5.3 & 0.23 \\
\hline \multicolumn{2}{|c|}{ T8: RP1.0 + VitE100 } & $6.5^{\mathrm{abc}}$ & 55.8 & 2.39 & 1130.0 & 76.7 & 4.3 & 8.3 & 0.35 \\
\hline \multicolumn{2}{|c|}{ T9: RP1.0 + VitE200 } & $6.9^{a, c}$ & 45.7 & 2.03 & 990.0 & 68.3 & 4.4 & 5.3 & 0.24 \\
\hline
\end{tabular}

$a, b, c:$ Different letters within the same column indicate significant differences among treatment groups $(p<0.05)$.

N.S. - Not significant. 
the starter period (weeks 1-3), representing the early adaptation period of the immature gut, a significant RP $X$ VitE interaction was observed, as confirmed by the differences in feed intake between T7 and T9 groups. This period is very important because small intestine growth peaks around the final of first week up to the early $3^{\text {rd }}$ week post-hatch (Uni et al., 1999; Noy et al., 2001). It was shown that high VitE levels in excess of nutritional requirements may reduce oxidative stress (Mcllroy et al., 1993), which may explain the RP X VitE interactions observed in our study, when high RP levels presumably caused stress in broilers.

In present study, both RP and VitE additives influenced plasma uric acid concentrations: 0.5\% RP reduced uric acid levels compared with 1.0\% RP (3.9vs. $5.8 \pm 0.5 \mathrm{mg} / \mathrm{dL}$, respectively). This is in agreement with Ghazalah \& Ali (2008), who observed reduction in blood uric acid level in broilers fed $0.5 \%$ rosemary leaves; however, VitE was not tested in their study. In our case, the supplementation of $100 \mathrm{mg}$ of VitE reduced plasma uric acid level to $3.9 \pm 0.5 \mathrm{mg} / \mathrm{dL}$, but further research with a higher number of birds is need in order to confirm this result.

In the present study, gut gross morphology was also affected RP and VitE additives. Duodenal width was positively affected both by $1.0 \% \mathrm{RP}$ and $200 \mathrm{mg}$ VitE levels, as well as their interaction. Additionally, poor development of the jejunum was observed at dietary 0.5 and $1.0 \%$ RP levels. Groups T2 and T4 showed lower jejunal width $(4.2 \mathrm{~mm}$ and $4.6 \mathrm{~mm}$ ) than group T1 $(7.0 \mathrm{~mm})$, but higher values were obtained group T9 $(6.9 \pm 0.5 \mathrm{~mm})$ than group T4. These differences evidence an interaction between both additives added at high levels, suggesting the protective role of VitE at $200 \mathrm{mg}$ level when $1 \%$ of RP was added. In fact, a positive effect of VitE and glutamine, supplemented until one week post hatch, on duodenum development was observed by Murakami et al. (2007).

In our study, large intestine (cecum and colon) morphometry was negatively affected only by $1.0 \%$ $\mathrm{RP}$, whereas $0.5 \% \mathrm{RP}$ increased right cecum relative weight. The cecum is an important site of bacterial digestion, and the colon physiology is influenced by dietary fiber contents. The positive effect of $0.5 \% \mathrm{RP}$ on broiler performance observed in the present study may be justified by a positive effect on cecal bacteria, similar to that obtained with subtherapeutic doses of antibiotics (Mathlouthi et al., 2012). Moreover, the negative influence of RP on small intestine, particularly at $1.0 \%$, may also partially explain the variations in plasma protein and uric acid levels. In fact, the duodenum, jejunum and ileum are the main organs responsible for nutrient digestion and absorption (Murakami et al., 2007). Unfortunately, the intestinal microflora and histological traits were not evaluated in the present study and further research is needed to elucidate this effect.

Ghazalah \& Ali (2008) did not observe any effects of feeding broilers with rosemary leaves on broiler carcass, heart, liver, and abdominal fat relative weights, except for the gizzard, which was lighter compared with the control group. Although the gut morphometry was affected by both additives, in the present study, the other evaluated organs and tissues, as well as carcass parts, were not influenced by the treatments.

We conclude that the dietary supplementation levels $0.5 \%$ RP and $200 \mathrm{mg} / \mathrm{kg}$ VitE improved broiler performance, and did not cause any evident adverse effects. Gut gross morphometry was negatively affected by the dietary supplementation of $1.0 \%$ RP. High rosemary powder levels in the diet may impair broiler performance, despite the potential protective role of vitamin $\mathrm{E}$.

\section{ACKNOWLEDGMENTS}

The financial support by Rasht Branch, Islamic Azad University, grant number 4.5830 is gratefully acknowledged.

\section{REFERENCES}

Abdullah AY, Mahmoud KZ, Nusairat BM, Qudsieh RI. Small intestinal histology, production parameters, and meat quality as influenced by dietary supplementation of garlic (Allium sativum) in broiler chicks. Italian Journal of Animal Science 2010;9:e80.

AOAC. Official methods of analysis. 18th ed. Washington; 2005

Applegate TJ, Dibner JJ, Kitchell ML, Uni Z, Lilburn MS. Effect of turkey (Meleagridis gallopavo) breeder hen age and egg size on poult development. 2. Intestinal villus growth, enterocyte migration and proliferation of the turkey poult. Comparative Biochemistry and Physiology Part B: Biochemistry and Molecular Biology 1999;124:381389.

Charles DJ. Rosemary. In: Charles DJ, editor. Antioxidant properties of spices, herbs and other sources. New York: Springer; 2013. p.495-507.

Franz C, Baser K, Windisch W. Essential oils and aromatic plants in animal feeding - a European perspective. A review. Flavour and Fragrance Journal 2010;25:327-340.

García V, Catalá-Gregori P, Hernández F, Megías MD, Madrid J. Effect of formic acid and plant extracts on growth, nutrient digestibility, intestine mucosa morphology, and meat yield of broilers. Journal of Applied Poultry Research 2007;16:555-562.

Ghazalah AA, Ali AM. Rosemary leaves as a dietary supplement for growth in broiler chickens. International Journal of Poultry Science 2008;7:234239. 
Giannenas I, Papaneophytou CP, Tsalie E, Pappas I, Triantafillou E, Tontis $D$, et al. Dietary supplementation of benzoic acid and essential oil compounds affects buffering capacity of the feeds, performance of turkey poults and their antioxidant status, $\mathrm{pH}$ in the digestive tract, intestinal microbiota and morphology. Asian-Australasian Journal of Animal Sciences 2014;27:225-236.

Hashemi SR, Davoodi H. Herbal plants and their derivatives as growth and health promoters in animal nutrition. Veterinary Research Communications 2011;35:169-180.

Hernández F, Madrid J, García V, Orengo J, Megías MD. Influence of two plant extracts on broilers performance, digestibility, and digestive organ size. Poultry Science 2004;83:169-174.

Ibañez E, Kubátová A, Señoráns FJ, Cavero S, Reglero G, Hawthorne SB. Subcritical water extraction of antioxidant compounds from rosemary plants. Journal of Agricultural and Food Chemistry 2003;51:375-382.

Khan RU, Rahman ZU, NikousefatZ, Javdani M, Tufarelli V, Dario C, et al. Immunomodulating effects of vitamin $\mathrm{E}$ in broilers. World's Poultry Science Journal 2012;68:31-40.

Lokaewmanee K, Yamauchi K, Thongwittaya N. Effects of fermented plant product on growth performance, some blood variables, carcass characteristics, and intestinal histology in broilers. British Poultry Science 2012;53:215-223.

Lu T, Harper AF, Zhao J, Corl BA, LeRoith T, Dalloul RA. Effects of a dietary antioxidant blend and vitamin $E$ on fatty acid profile, liver function, and inflammatory response in broiler chickens fed a diet high in oxidants. Poultry Science 2014a;93:1658-1666.

Lu T, Harper AF, Zhao J, Dalloul RA. Effects of a dietary antioxidant blend and vitamin $\mathrm{E}$ on growth performance, oxidative status, and meat quality in broiler chickens fed a diet high in oxidants. Poultry Science 2014b;93:1649-1657.

Mathlouthi N, Bouzaienne T, Oueslati I, Recoquillay F, Hamdi M, Urdaci M, et al. Use of rosemary, oregano, and a commercial blend of essential oils in broiler chickens: in vitro antimicrobial activities and effects on growth performance. Journal of Animal Science 2012;90:813-823.

Mcllroy SG, Goodall EA, Rice DA, McNulty MS, Kennedy DG. Improved performance in commercial flocks with subclinical infectious bursal disease when fed diets containing increased concentrations of vitamin E. Avian Pathology 1993;22:81-94.
Moreno S, Scheyer T, Romano CS, Vojnov AA. Antioxidant and antimicrobial activities of rosemary extracts linked to their polyphenol composition. Free Radical Research 2006;40:223-231.

Murakami AE, Sakamoto MI, Natali MR, Souza LM, Franco JR. Supplementation of glutamine and vitamin $\mathrm{E}$ on the morphometry of the intestinal mucosa in broiler chickens. Poultry Science 2007;86:488495

Noy Y, Geyra A, Sklan D. The effect of early feeding on growth and small intestinal development in the posthatch poult. Poultry Science 2001;80:912-919.

Polat U, Yesilbag D, Eren M. Serum biochemical profile of broiler chickens fed diets containing rosemary and rosemary volatile oil. Journal of Biological \& Environmental Sciences 2011;5:23-30

SPSS. SPSS base 7.5 for windows. Chicago; 1997

Uni Z, Noy Y, Sklan D. Posthatch development of small intestinal function in the poult. Poultry Science 1999;78:215-222

Windisch W, Schedle K, Plitzner C, Kroismayr A. Use of phytogenic products as feed additives for swine and poultry. Journal of Animal Science 2008:86(14 Suppl):E140-E148

Xiao R, Power RF, Mallonee D, Crowdus C, Brennan KM, Ao T, et al. A comparative transcriptomic study of vitamin $\mathrm{E}$ and an algae-based antioxidant as antioxidative agents: investigation of replacing vitamin $\mathrm{E}$ with the algae-based antioxidant in broiler diets. Poultry Science 2011;90:136-146

Yesilbag D, Eren M, Agel H, Kovanlikaya A, Balci F. Effects of dietary rosemary, rosemary volatile oil and vitamin $\mathrm{E}$ on broiler performance, meat quality and serum SOD activity. British Poultry Science 2011;52:472-482.

Yesilbag D, Gezen SS, Biricik H, Bulbul T. Effect of a rosemary and oregano volatile oil mixture on performance, lipid oxidation of meat and haematological parameters in Pharaoh quails. Poultry Science 2012;53:89-97

Yesilbag D, Gezen SS, Biricik H, Meral Y. Effects of dietary rosemary and oregano volatile oil mixture on quail performance, egg traits and egg oxidative stability. British Poultry Science 2013:54:231-237. 